Recepción: 07 / 10/ 2018

Aceptación: 20 / 11 / 2018

(c) 1 (1) (2)

Ciencias de la salud

Publicación: 20 / 01 / 2019

Artículo de Revisión

\title{
Tratamiento en pacientes con insuficiencia renal crónica
}

\author{
Treatment in patients with chronic renal failure
}

Tratamento em pacientes com insuficiência renal crónica

Angela R. Briones-Mera ${ }^{\mathrm{I}}$

arbriones@utm.edu.ec

Miguel A. Alvarez-Menéndez II

alvarezmd2010@hotmail.com

Mónica P. Mastarreno-Cedeño III

mmastarreno@utm.edu.ec

Dolores I. Chavarria-Cedeño IV

Isadol1975@hotmail.com

Reina Y. Villavicencio-Macías V

rvillavicencio@utm.edu.ec

Marcos A. Córdova-Muñiz VI

marcm_9124@hotmail.com

Correspondencia: arbriones@utm.edu.ec

I. Licenciada en Enfermería; Maestría en Investigación Clínica y Epidemiológica; Docente de la Universidad Técnica de Manabí, Manabí, Ecuador.

II. Médico Cirujano; Medico de la Unidad de Bienestar Estudiantil de la UNESUM, Jipijapa, Manabí, Ecuador.

III. Magíster en Docencia Universitaria; Docente de la Universidad Técnica de Manabí, Jipijapa, Manabí, Ecuador.

IV. Especialista en gerencia en Salud; Universidad Técnica de Manabí. Jipijapa, Manabí, Ecuador.

V. Licenciada en Enfermería; Docente en la Universidad Técnica de Manabí, Jipijapa, Manabí, Ecuador.

VI. Licenciado en Enfermería en la Práctica Privada, Docente en la Universidad Técnica de Manabí, Jipijapa, Manabí, Ecuador. 


\title{
Resumen
}

La Enfermedad Renal Crónica (ERC) es un problema de salud pública importante, es una patología rara y compleja, pero que en sus fases tempranas es frecuente y de fácil tratamiento. El gran espectro clínico de presentación de la ERC varía, desde hallazgos de laboratorio asintomáticos hasta presentaciones extremas con fracturas óseas y deterioro cognitivo. La expectativa de vida a nivel mundial y regional ha aumentado progresivamente. En el Ecuador, ha sucedido algo similar, y las proyecciones tienden al alza (Tanto los determinantes sociales del desarrollo de ERC como la forma de atención se producen de manera diferencial entre los distintos grupos sociales. Globalmente, las personas más susceptibles de adquirir la ERC tienen condiciones estructurales asimétricas relacionadas con sus modos de vida. Esta revisión bibliográfica contiene aspectos técnicos estudiados alrededor de los resultados experimentales de los tipos de tratamientos indicados solo para pacientes con enfermedad en estado avanzado o crítico, incluyendo algunas reflexiones respecto a la posición del Estado y las condiciones psicosociales del paciente y su núcleo familiar. No hay que olvidar la importancia de este padecimiento porque este proceso patológico es una causa importante de muerte, tampoco la discapacidad severa de nuestros pacientes. Es necesario tener en cuenta el papel que juega el primer nivel de atención en el tratamiento.

Palabras claves: Insuficiencia Renal; Hipertensiva; Nefropatía Diabética; Crónica.

\begin{abstract}
Chronic Kidney Disease (CKD) is an important public health problem, it is a rare and complex pathology, but in its early stages it is frequent and easy to treat. The large clinical spectrum of presentation of $\mathrm{CKD}$ varies, from asymptomatic laboratory findings to extreme presentations with bone fractures and cognitive impairment. Life expectancy worldwide and regionally has increased progressively. In Ecuador, something similar has happened, and the projections tend to rise (both the social determinants of the development of CKD and the way of care occur differentially among different social groups.) Overall, the people most likely to acquire CKD have asymmetric structural conditions related to their lifestyles This bibliographic review contains technical aspects studied around the experimental results of the types of treatments indicated only for patients with advanced or critical illness, including some reflections regarding the position of the State and The psychosocial conditions of the patient and his / her family nucleus must not be forgotten, because
\end{abstract}


Angela R. Briones-Mera; Miguel A. Alvarez-Menéndez; Mónica P. Mastarreno-Cedeño; Dolores I. Chavarria-Cedeño; Reina Y. Villavicencio-Macías; Marcos A. Córdova-Muñiz

the pathological process is an important cause of death, and the severe disability of our patients must not be overlooked. Of attention in the treatment.

Keys words: Renal insufficiency; Hypertensive; Diabetic nephropathy; Chronicle.

\section{Resumo.}

A Doença Renal Crônica (DRC) é um importante problema de saúde pública, é uma patologia rara e complexa, mas em seus estágios iniciais é freqüente e de fácil tratamento. O amplo espectro clínico de apresentação da DRC varia de achados laboratoriais assintomáticos a apresentações extremas com fraturas ósseas e comprometimento cognitivo. A expectativa de vida em todo o mundo e regionalmente aumentou progressivamente. No Equador, algo semelhante aconteceu e as projeções tendem a aumentar (tanto os determinantes sociais do desenvolvimento da DRC quanto o modo de cuidar ocorrem diferencialmente entre os diferentes grupos sociais). No geral, as pessoas com maior probabilidade de adquirir DRC têm condições estruturais assimétricas. Relacionados a seus estilos de vida Esta revisão bibliográfica contém aspectos técnicos estudados em torno dos resultados experimentais dos tipos de tratamentos indicados apenas para pacientes com doença avançada ou crítica, incluindo algumas reflexões sobre a posição do Estado e as condições psicossociais do paciente e de seus pacientes. o núcleo familiar não deve ser esquecido, porque o processo patológico é uma importante causa de morte, e a grave incapacidade de nossos pacientes não deve ser negligenciada. De atenção no tratamento.

Palavras chaves: Insuficiência renal; Hipertensivo; Nefropatia diabética; Crônica.

\section{Introducción.}

La Enfermedad Renal Crónica (ERC) es un problema de salud pública importante, es una patología rara y compleja, pero que en sus fases tempranas es frecuente y de fácil tratamiento. Sólo una pequeña proporción de enfermos evoluciona hacia la insuficiencia renal terminal con sus complicaciones asociadas y necesidad de tratamiento renal sustitutivo. Esta evolución hacia la pérdida de función renal tiene un curso progresivo, en el que podemos influir mediante una actuación precoz sobre sus principales causas: hipertensión arterial (HTA) y diabetes mellitus. El 
control de estas dos afecciones debe ser estricto y adecuado a las recomendaciones de las guías en vigor, no sólo para minimizar su progresión y tratar las complicaciones inherentes a la insuficiencia renal, sino también para reducir el riesgo vascular asociado a la ERC. El descenso de la función renal se asocia de forma significativa con un mayor riesgo cardiovascular. (Egocheaga, Lobos, \& Alvarez Guissasol, 2007)

El gran espectro clínico de presentación de la ERC varía, desde hallazgos de laboratorio asintomáticos hasta presentaciones extremas con fracturas óseas y deterioro cognitivo. Esta variedad ha determinado que en muchos casos la ERC sea sub diagnosticada y sub tratada, evolucionando a estadios finales en los cuales el tratamiento es exclusivamente sustitutivo más no curativo. El sub diagnóstico, sub registro y sub tratamiento conllevan a un incremento de la morbi-mortalidad y altos costos para el Estado, consecuencias que podrían ser prevenidas, retardadas y disminuidas tan solo con la identificación precoz de la ERC. (Ministerio de Salud Pública, 2018)

El riesgo de compromiso renal se incrementa debido a factores ambientales desfavorables, incluyendo la privación y la malnutrición en el embarazo, exposición a metales pesados, contaminantes en la agricultura, sustancias herbolarias y trabajo en climas de calor extremo. Aparte de condiciones que predisponen a la ERC (diabetes, obesidad, hipertensión, patologías glomerulares y tubulointersticiales) (Ministerio de Salud Pública, 2018)

Aspectos relevantes sobre la ERC en el Ecuador

La expectativa de vida a nivel mundial y regional ha aumentado progresivamente. En el Ecuador, ha sucedido algo similar, y las proyecciones tienden al alza (gráfico 1). La importancia de esta tendencia radica en el aumento de casos nuevos de ERC y el empeoramiento de los casos establecidos, con el consecuente aumento en el gasto público. 
Angela R. Briones-Mera; Miguel A. Alvarez-Menéndez; Mónica P. Mastarreno-Cedeño; Dolores I. Chavarria-Cedeño; Reina Y. Villavicencio-Macías; Marcos A. Córdova-Muñiz

Gráfico $N^{\circ} 1 .-$ Expectativa de vida global en el Ecuador 1990-2015

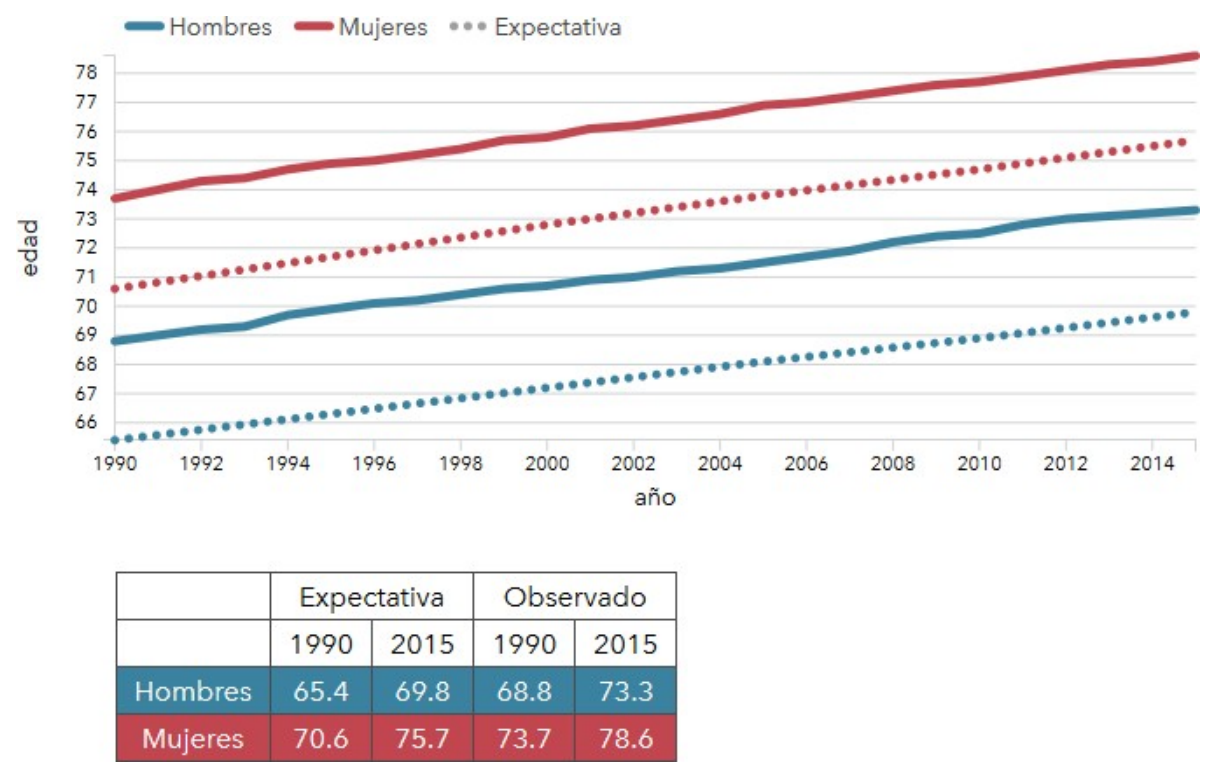

Tomado de: Institute of Health Metrics and Evaluation, 2015.(26)

Si se considera la tendencia al aumento de la expectativa de vida, junto a las estimaciones y proyecciones de la SLANH y la NANHES III, es posible establecer la población nacional aproximada afectada por la ERC de acuerdo con su respectivo estadio (tabla 1). 


\section{Grafico $N^{\circ}$ 2.- Principales causas de años de vida ajustados por discapacidad y cambio}

porcentual 2005-2015

Enfermedades transmisibles, maternas, neonatales y de la nutrición

Enfermedades no transmisibles

Lesiones

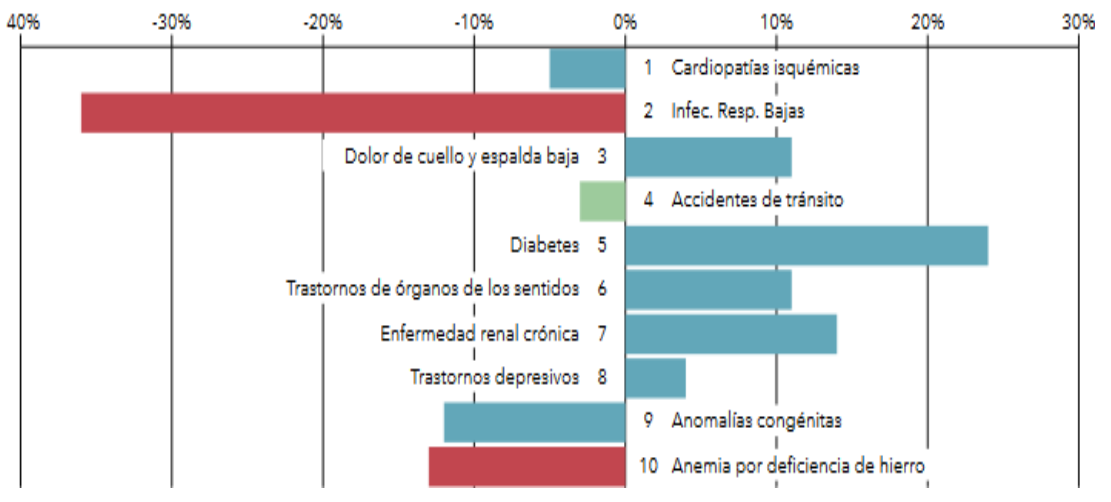

En términos en años de vida perdida por muerte prematura (AVPP), ha sucedido algo similar en el Ecuador, donde se aprecia una tendencia a la preponderancia de enfermedades crónicas no transmisibles, incluyendo a la ERC.

Tanto los determinantes sociales del desarrollo de ERC como la forma de atención se producen de manera diferencial entre los distintos grupos sociales. Globalmente, las personas más susceptibles de adquirir la ERC tienen condiciones estructurales asimétricas relacionadas con sus modos de vida. Estos diferenciales implican no solo una condición de vulnerabilidad diferente para enfermar por ERC, sino que las personas afectadas también soportan conductas de inequidad en la prestación de servicios de salud y el acceso a los tratamientos. Ambos tipos de diferenciales se presentan en virtud de la raza, la zona geográfica y la condición social. (Lopera-Medina, 2016) 
Angela R. Briones-Mera; Miguel A. Alvarez-Menéndez; Mónica P. Mastarreno-Cedeño; Dolores I. Chavarria-Cedeño; Reina Y. Villavicencio-Macías; Marcos A. Córdova-Muñiz

\section{Metodología.}

Se ha considerado un número suficiente de publicaciones científicas respecto a la enfermedad renal crónica, combinando los aspectos relevantes de cada publicación direccionados al tratamiento y seguimiento evolutivo de la misma.

Por tanto, esta revisión bibliográfica contiene aspectos técnicos estudiados alrededor de los resultados experimentales de los tipos de tratamientos indicados solo para pacientes con enfermedad en estado avanzado o crítico, incluyendo algunas reflexiones respecto a la posición del Estado y las condiciones psicosociales del paciente y su núcleo familiar.

\section{Resultados.}

Los problemas que pueden causar falla renal pueden incluir principalmente la diabetes, le siguen la Hipertensión arterial, enfermedades autoinmunes, como el lupus y la nefropatía IgA, enfermedades genéticas como la enfermedad renal poliquística, Síndrome nefrótico, problemas de vías urinarias. La falla o insuficiencia renal aguda sucede cuando los riñones dejan de funcionar de repente por al menos 2 días., las causas incluyen: Ataque de corazón, uso ilegal de drogas y el abuso de drogas, insuficiente flujo de sangre a los riñones o problemas de las vías urinarias. Estas fallas pueden que no sean permanentes, los riñones Vivir una vida saludable y trabajando con el medico te puede ayudar a controlar estos problemas de salud y puede ayudar que los riñones funcionen lo más largo posible. La falla renal, también llamada enfermedad renal de etapa termina (ERET o ESRD por sus siglas en ingles), es la última etapa de la enfermedad renal crónica; esto quiere decir que han parado de trabajar suficiente para sobrevivir sin el tratamiento de diálisis o un trasplante de riñón. (AKF, 2019) 
La progresión de la ERC puede ser acelerada por enfermedades intercurrentes, el uso de agentes nefrotóxicos, un mal control dietético-metabólico, un inadecuado control de la hipertensión arterial y la superposición de episodios de injuria renal aguda que actualmente se la ha considerado como al equivalente de una cardiopatía isquémica tipo infarto agudo de miocardio por algunos autores. Los pacientes con ERC en estadio 3 tienden a una disminución progresiva del filtrado glomerular cuando su condición se asocia a incremento de la albuminuria. Las personas en este estadio empiezan a expresar alteraciones sistémicas secundarias a su ERC (por ejemplo, trastornos del metabolismo calcio-fósforo). (Hurtado-Aréstegui, 2016)

En la enfermedad renal crónica avanzada (ERCA) que incluye a los estadios 4 y 5 , se hacen más evidentes las manifestaciones clínicas como consecuencia del mayor deterioro de la función renal (anemia, hipertensión, sobrecarga hídrica) cuyo concepto extremo es lo que se conoce como síndrome urémico. Debido al pobre pronóstico de las personas con ERCA, al grupo de pacientes en estadio 5 que no han fallecido en todo el curso de la enfermedad por las complicaciones cardiovasculares propias de su patología de base, se los ha llegado a considerar como “sobrevivientes”. (Ministerio de Salud Pública, 2018)

En general, todos los cambios a nivel renal, producidos por el envejecimiento, repercuten en la capacidad de soporte y respuesta del riñón a cualquier tipo de daño o injuria, aumentando la susceptibilidad a afectaciones agudas y a enfermedad renal crónica progresiva. Como desenlace, las personas con ERCA, frecuentemente se encuentran en un estado de intercambio de terapias, es decir de hemodiálisis a diálisis peritoneal o trasplante renal y viceversa hasta agotar todas las opciones de sustitución renal. Aún con tratamiento, las personas con ERCA presentan un deterioro clínico y progresivo de su estado general hasta terminar falleciendo como consecuencia de las complicaciones cardiovasculares e infecciosas. (Lopera-Medina, 2016) 
Angela R. Briones-Mera; Miguel A. Alvarez-Menéndez; Mónica P. Mastarreno-Cedeño; Dolores I. Chavarria-Cedeño; Reina Y. Villavicencio-Macías; Marcos A. Córdova-Muñiz

En relación con los mecanismos de progresión de la insuficiencia renal crónica se debe mencionar que una vez que la causa primaria ha ocasionado destrucción de un numero de nefronas se pondrán en marcha mecanismos que tratarán de remplazar la función de las nefronas destruidas, como consecuencia se produce hipertrofia e hiperfiltración de los glomérulos restantes que si no se corrige terminarán por destruirlos progresivamente. De los dos tipos de diálisis, la más utilizada es la hemodiálisis (HD) alcanzando un 80 a 90\%. La diálisis peritoneal continua ambulatoria (DPCA) se utiliza en un 10 a 20\%, con algunas excepciones. En la elección del tipo de diálisis usualmente se toma en cuenta factores como enfermedades coexistentes, situaciones vitales y sociales de cada paciente y también información de la comunidad nefrológica de las diferentes técnicas. Otros factores a considerar son preferencia del paciente y de la familia, capacidad de efectuar el procedimiento técnico en términos de seguridad y eficacia, costos, limitaciones anatómicas como hernias, lesiones vertebrales y limitaciones fisiológicas como el transporte peritoneal. (LoperaMedina, 2016)

Se considera una diálisis adecuada cuando el porcentaje de urea sérica extraída supera el $70 \%$ de la cifra de urea sérica prediálisis o cuando el aclaramiento de urea basado en modelos cinéticos de urea $(\mathrm{Kt} / \mathrm{v})$ es superior a 1,2. Recientemente, en vías de experimentación, se están realizando hemodiálisis diaria con excelentes resultados: mejor hematocrito, mejor control de la presión arterial, de la nutrición, del estado mental, función social, menor morbilidad y por lo tanto menor necesidad de hospitalización. El trasplante renal desde un comienzo se ha considerado el tratamiento de elección, por diferentes razones todas ellas válidas. La donación puede ser de una persona viva o de cadáver. La elección depende de factores culturales, socioeconómicos, legales, religiosos. (Lopera-Medina, 2016) 
Se ha señalado que el control de la presión arterial, de la glicemia en pacientes diabéticos y la dieta proteica es indispensable para lograr tal fin. La variedad de agentes hipotensores efectivos es enorme, sin embargo hay datos experimentales y clínicos que señalan que el uso de inhibidores de la enzima convertidora de la angiotensina o los bloqueadores de la angiotensina II, tiene ventajas comparado con otros agentes hipotensores. Incluso se está recomendando el uso de bloqueadores ECA aún en enfermos normotensos con enfermedad renal proteinúrica (Torres Zamudio, 2003)

Además se ha observado que la angiotensina II es un importante modulador de citoquinas inflamatorias y fibrogénicas. La angiotensina II también tiene un importante rol en el crecimiento e hipertrofia tisular y se interrelaciona con varias citoquinas que estimulan el crecimiento. Finalmente la habilidad de la angiotensina II para generar moléculas oxígeno reactivas, mediante activación del sistema fosfato de nicotinamida-adenina dinucleotido (NADP)/la forma reducida del NADP (NADPH), después de ligarse al receptor de la angiotensina sugiere un rol en crear stress oxidante en el tejido vascular. En relación a la aldosterona, últimamente se ha establecido que dicha hormona participaría en el proceso de fibrogénesis del tejido vascular y renal y de ser así se abrirían nuevas posibilidades terapéuticas para mejorar la evolución de pacientes con enfermedad renal crónica. Varios ensayos clínicos recientemente terminados han puesto de manifiesto la necesidad de inhibir el sistema renina-angiotensina-aldosterona. (Lopera-Medina, 2016)

La importancia de la genética para ayudarnos a entender mejor los beneficios de los agentes que inhiben el sistema renina-angiotensina-aldosterona puede proporcionarnos medidas terapéuticas más eficaces que no sólo retarden el progreso del daño renal sino que induzcan la regresión o remisión de la enfermedad renal crónica. Anteriormente se consideraba que la hiperuricemia era sólo un marcador de disfunción renal, pero recientemente se vienen realizando estudios que parecen demostrar que la hiperuricemia juega un rol en la progresión de la enfermedad renal, agravando la 
Angela R. Briones-Mera; Miguel A. Alvarez-Menéndez; Mónica P. Mastarreno-Cedeño; Dolores I. Chavarria-Cedeño; Reina Y. Villavicencio-Macías; Marcos A. Córdova-Muñiz

hipertensión arterial, la proteinuria y la insuficiencia renal. Si bien la mayoría de los estudios que muestran que la hiperuricemia es un factor agravante de la insuficiencia renal se han realizado en animales de experimentación, se espera que lo mismo suceda en seres humanos, por lo tanto la recomendación es utilizar el allopurinol para normalizar los niveles séricos de ácido úrico. (LoperaMedina, 2016)

Para terminar es importante mencionar a las quimokinas, que son cytokinas de bajo peso molecular y a los receptores de quimokinas, que según estudios experimentales y observaciones clínicas parecen estar involucrados en la resolución o progresión de enfermedad renal. Hasta la fecha se han reconocido más de 44 quimokinas y 24 receptores de quimokinas. El uso de antagonistas de los receptores de quimokinas se presenta como arma terapéutica de gran potencial en el tratamiento de la insuficiencia renal progresiva. (Torres Zamudio, 2003)

Esta somera revisión del tema de la insuficiencia renal crónica ha puesto de manifiesto cuan complejo y numerosos son los factores que participan en la patogénesis de la insuficiencia renal crónica pero al mismo tiempo nos hace ver cuán promisorio es la posibilidad de encontrar procedimientos terapéuticos que eviten, aminoren y en última instancia resuelvan exitosamente el curso de la insuficiencia renal.

Las desigualdades en salud en el caso de la ERC representan inequidades porque, al tratarse principalmente de problemas de salud crónicos, con frecuencia están asociados con deficiente manejo por parte del sistema de salud, lo que, por lo general, afecta a menudo a los grupos con menor acceso a los servicios de salud y menor calidad en ellos. Se ha informado que las personas en edad productiva, las mujeres, los mayores de sesenta años y las personas más pobres tienen mayores dificultades para acceder al sistema de salud y, por lo tanto, padecen discriminación y exclusión. 
Estos diferenciales, por ser injustos e inaceptables, revelan inequidades sociales y sanitarias. Para ilustrar estas inequidades puede citarse el estudio de Martínez, en el cual se incluyeron pacientes diabéticos que padecieron ERC-5. Este estudio encontró que los pacientes pertenecían predominantemente a grupos con bajo nivel educativo y bajo estrato socioeconómico. Adicionalmente, se encontraron diferencias en la calidad de la atención en las poblaciones del régimen subsidiado. Algunas investigaciones en el ámbito mundial refuerzan este hallazgo, en la medida en que han encontrado asociación entre la ERC y el bajo nivel de información en salud. (Lopera-Medina, 2016)

\section{Conclusiones.}

El desafío y complejidad que representa la gestión de la enfermedad renal crónica, ha provocado que varias instituciones científicas de diferente naturaleza hayan desarrollado guías de práctica clínica (GPC) para el manejo óptimo de la ERC, con base en un riguroso proceso de revisión sistemática de la evidencia disponible. (Torres Zamudio, 2003)

La ERC es la cuarta causa de mortalidad general y la quinta de mortalidad prematura en el Ecuador. La mortalidad por ERC en el Ecuador alcanza niveles entre el $6 \%$ y 7 \%. El 1,44 \% de años vividos con discapacidad son producidos por la ERC en el Ecuador, aunque la esperanza de vida corregida por discapacidad indica 3,47 \%. En países desarrollados, la ERC constituye la patología más frecuente y que mayores costos genera dentro de los programas de salud pública. Se estima que en el Ecuador existen cerca de diez mil personas en tratamiento con hemodiálisis y diálisis peritoneal, lo que representa una tasa de 660 casos por millón de habitantes. En estos pacientes el período de supervivencia promedio es de 52 meses, un equivalente inferior a 5 años. (Ministerio de Salud Pública, 2018) 
Angela R. Briones-Mera; Miguel A. Alvarez-Menéndez; Mónica P. Mastarreno-Cedeño; Dolores I. Chavarria-Cedeño; Reina Y. Villavicencio-Macías; Marcos A. Córdova-Muñiz

La complejidad de la situación de la ERC en un contexto de cambios globales en el mundo contemporáneo amerita instaurar mecanismos de salud pública que prevengan, traten y mitiguen el impacto de esta enfermedad. Ello implica trascender los factores de riesgo individuales, para reconocer la influencia de complejos fenómenos sociales y, por lo tanto, implementar cambios paradigmáticos que ofrezcan una mejor respuesta para su manejo.

La planificación sanitaria debe llevar al mejoramiento de los resultados en salud, entendidos como la reducción de la incidencia, de la mortalidad y de la discapacidad, así como la mitigación del impacto psicosocial y económico que se genera en los pacientes afectados. Para ello, es necesario efectuar un cambio de modelo de atención por uno más comprehensivo e integral, que permita impactar en los determinantes de la salud y los modos de vida, reducir los riesgos y garantizar el manejo integral de la enfermedad como un proceso que amerita oportunidad y calidad. (LoperaMedina, 2016)

Tomando en cuenta las estimaciones de la Sociedad Latinoamericana de Nefrología e Hipertensión (SLANH) y de la Tercera Encuesta de Salud y Nutrición (NANHES III por sus siglas en inglés), en el Ecuador, se estima que aproximadamente un $45 \%$ de pacientes en estadios 4 y 5 podrían fallecer antes de iniciar tratamiento con diálisis. Solo en estadio 5, se sabe que en el Ecuador existirían más de 30000 personas afectadas (Ministerio de Salud Pública, 2018)

En el Ecuador, además de su impacto epidemiológico, la ERC produce grandes problemas derivados de la atención en salud. Entre los principales están: referencia tardía o en estadios avanzados, ausencia de programas de identificación precoz en personas con factores de riesgo para ERC, falta de médicos especialistas, limitación de opciones terapéuticas para controlar de mejor 
manera las complicaciones de la ERC y ausencia de documentos normativos claros enfocados en los pacientes con enfermedad renal. (Ministerio de Salud Pública, 2018)

En años recientes, el concepto de enfermedad renal crónica ha estado sometido a la tensión de un cambio de paradigma; esto es acerca de la perspectiva básica de ERC, que se ha desviado, desde la falla renal como una enfermedad que amenaza la vida, hacia las etapas precoces de ERC, como el objetivo clave para su prevención, detección, evaluación y manejo. Los gobiernos deben adoptar una política pública relacionada a ERC. ERC es un componente clave de un grupo de enfermedades crónicas, que incluye la hipertensión, diabetes y enfermedad cardiovascular; para optimizar recursos, ERC debe ser integrada a las políticas públicas existentes de estas enfermedades. Todos los países deberían elaborar estrategias para reducir gradualmente el consumo de sal en la población. (Carlos Flores, 2010)

No hay que olvidar la importancia de este padecimiento porque este proceso patológico es una causa importante de muerte, tampoco la discapacidad severa de nuestros pacientes. Es necesario tener en cuenta el papel que juega el primer nivel de atención en el tratamiento. (Ávila-Saldiva, 2013)

\section{Bibliografía.}

AKF. (2019). Falla renal. American Kidney Fund: Rockville Pike. Recuperado el 1 de 2019, de http://www.kidneyfund.org/en-espanol/enfermedad-de-los-rinones/falla-de-los-rinones/

Ávila-Saldiva, M. (2013). Enfermedad renal crónica: prevención y detección temprana en el primer nivel de atención. Medicina Interna de México, No. 2 , 148-153.

Carlos Flores, H. J. (2010). Enfermedad renal crónica: epidemiología y factores de riesgo . Revista Médica Clínica Las Condes, 500-664. 
Angela R. Briones-Mera; Miguel A. Alvarez-Menéndez; Mónica P. Mastarreno-Cedeño; Dolores I. Chavarria-Cedeño; Reina Y. Villavicencio-Macías; Marcos A. Córdova-Muñiz

Egocheaga, M. I., Lobos, J. M., \& Alvarez Guissasol, F. (2007). Documento de consenso sobre la enfermedad renal crónica. Sociedad Española de Medicina de Familia y Comunitaria y Sociedad Española de Nefrología. Barcelona: Semfyc Ediciones.

Hurtado-Aréstegui, A. (2016). Manejo de la enfermedad renal crónica. Management of the chronic renal disease, 19(2), 50-54.

Lopera-Medina, M. M. (2016). La enfermedad renal crónica en Colombia: necesidades en salud y respuesta del Sistema General de Seguridad Social en Salud. Revista Gerencia y Políticas de Salud, 212-233.

Ministerio de Salud Pública. (2018). Prevención, diagnóstico y tratamiento de la enfermedad renal crónica. Guía de Práctica Clínica (GPC). Quito: Dirección Nacional deNormatización MSP.

Torres Zamudio, C. (2003). Insuficiencia renal crónica. Revista Médica Herediana, http://www.scielo.org.pe/scielo.php?script=sci_arttext\&pid=S1018-130X2003000100001. 